\title{
Article \\ The Effect of Implementing Mechanical Cardiopulmonary Resuscitation Devices on Out-of-Hospital Cardiac Arrest Patients in an Urban City of Taiwan
}

\author{
Yi-Rong Chen ${ }^{1,+}{ }^{\dagger}$ Chi-Jiang Liao ${ }^{1,+}{ }^{+}$, Han-Chun Huang ${ }^{1}$, Cheng-Han Tsai ${ }^{2}$, Yao-Sing Su ${ }^{3}$, Chung-Hsien Liu ${ }^{1}$, \\ Chi-Feng Hsu ${ }^{1, *, \ddagger(D)}$ and Ming-Jen Tsai ${ }^{1, *, \ddagger(D)}$ \\ 1 Department of Emergency Medicine, Ditmanson Medical Foundation Chia-Yi Christian Hospital, \\ Chiayi City 600, Taiwan; masterdoctorsky@yahoo.com.tw (Y.-R.C.); matthewstliao@yahoo.com.tw (C.-J.L.); \\ lastar0329@gmail.com (H.-C.H.); cych03283@gmail.com (C.-H.L.) \\ 2 Department of Emergency Medicine, Taichung Veteran's General Hospital, Chia-Yi Branch, \\ Chiayi City 600, Taiwan; chtsai6482@gmail.com \\ 3 Fire Bureau, Chiayi City Government, Chiayi City 600, Taiwan; fire001@ems.chiayi.gov.tw \\ * Correspondence: alcooltw@gmail.com (C.-F.H.); tshi33@gmail.com (M.-J.T.) \\ + These authors contributed equally to this work. \\ $\ddagger \quad$ These authors contributed equally to this work.
}

Citation: Chen, Y.-R.; Liao, C.-J.; Huang, H.-C.; Tsai, C.-H.; Su, Y.-S.; Liu, C.-H.; Hsu, C.-F.; Tsai, M.-J. The Effect of Implementing Mechanical Cardiopulmonary Resuscitation Devices on Out-of-Hospital Cardiac Arrest Patients in an Urban City of Taiwan. Int. J. Environ. Res. Public Health 2021, 18, 3636. https:// doi.org/10.3390/ijerph18073636

Received: 10 March 2021

Accepted: 29 March 2021

Published: 31 March 2021

Publisher's Note: MDPI stays neutral with regard to jurisdictional claims in published maps and institutional affiliations.

Copyright: (c) 2021 by the authors. Licensee MDPI, Basel, Switzerland. This article is an open access article distributed under the terms and conditions of the Creative Commons Attribution (CC BY) license (https:// creativecommons.org/licenses/by/ $4.0 /)$
Abstract: High-quality cardiopulmonary resuscitation (CPR) is a key element in out-of-hospital cardiac arrest (OHCA) resuscitation. Mechanical CPR devices have been developed to provide uninterrupted and high-quality CPR. Although human studies have shown controversial results in favor of mechanical CPR devices, their application in pre-hospital settings continues to increase. There remains scant data on the pre-hospital use of mechanical CPR devices in Asia. Therefore, we conducted a retrospective cohort study between September 2018 and August 2020 in an urban city of Taiwan to analyze the effects of mechanical CPR devices on the outcomes of OHCA; the primary outcome was attainment of return of spontaneous circulation (ROSC). Of 552 patients with OHCA, 279 received mechanical CPR and 273 received manual CPR, before being transferred to the hospital. After multivariate adjustment for the influencing factors, mechanical CPR was independently associated with achievement of any ROSC (OR $=1.871 ; 95 \% \mathrm{CI}: 1.195-2.930)$ and sustained ( $\geq 24 \mathrm{~h})$ ROSC (OR $=2.353 ; 95 \% \mathrm{CI}: 1.427-3.879)$. Subgroup analyses demonstrated that mechanical CPR is beneficial in shorter emergency medical service response time ( $\leq 4 \mathrm{~min})$, witnessed cardiac arrest, and non-shockable cardiac rhythm. These findings support the importance of early EMS activation and high-quality CPR in OHCA resuscitation.

Keywords: mechanical CPR device; out-of-hospital cardiac arrest; resuscitation; return of spontaneous circulation

\section{Introduction}

Out-of-hospital cardiac arrest (OHCA) is a global health issue. Each year, cardiac arrest accounts for up to 3.7 million lives worldwide [1]. The incidence of OHCA has been reported to be 86.4 per 100,000 person-years in Europe, 98.1 in North America, 52.5 in Asia, and 51.1 in Taiwan [2,3]. Sustained return of spontaneous circulation (ROSC) from OHCA relies on the integral chain of survival, including early activation of the emergency medical services (EMS) system, provision of high-quality cardiopulmonary resuscitation (CPR), early defibrillation, advanced resuscitation, post-cardiac-arrest care, and recovery [4]. High-quality CPR plays a crucial role in survival of cardiac arrest [4].

High-quality and high-performance CPR has been promoted for preserving brain perfusion following ROSC as well as favorable neurological outcomes. However, there are many obstacles in pre-hospital settings that prevent the EMS system from meeting the requirements of high-quality CPR: sufficient compression rate, adequate depth, chest wall 
full recoil, and prevention of interruption. On the other hand, for maximizing the quality of CPR, mechanical CPR devices have been widely implemented in both pre-hospital and in-hospital settings in order to provide sufficient compression rate, adequate depth, and prevent the physical fatigue and interruption caused by manual CPR. However, there is a lack of evidence in current studies suggesting that in-hospital use of mechanical $\mathrm{CPR}$ devices is superior to manual CPR for acquiring ROSC or preferred neurological outcomes [5-7].

In pre-hospital settings, the effect of mechanical cardiopulmonary devices on patient outcome remains controversial. Randomized control trials conducted in Europe and the United States showed no difference in survival compared to manual CPR [8-11]. However, the majority of observational studies, including a recent large-scale German study, showed that mechanical CPR was associated with an increased rate of ROSC and may improve survival to hospital admission [12,13]. Mannequin studies also showed that mechanical CPR devices had higher chest compression quality during pre-hospital ambulance transport $[14,15]$. Hence, despite no definitive evidence of survival benefit, the application of mechanical CPR devices to reduce physical and cognitive load on emergency medical technicians (EMTs) continues to increase [16]. The results of previous studies investigating pre-hospital use of mechanical CPR devices on the outcome of patients with OHCA is summarized in Supplementary Table S1.

There are still scarce data concerning the use of mechanical CPR devices in pre-hospital settings in Asia. This study, conducted in an urban city of Taiwan, aimed to evaluate the impact of implementing mechanical CPR devices on ROSC, sustained ROSC for more than $24 \mathrm{~h}$, and survival at discharge with favorable neurological outcomes in OHCA patients.

\section{Materials and Methods}

\subsection{Study Design and Settings}

We conducted a retrospective cohort study between September 2018 and August 2020 (2 years) to evaluate the effect of mechanical CPR devices on the outcome of OHCAs in Chiayi City, Taiwan. All patients with OHCA who activated the EMS system were included in the OHCA registry of Chiayi City, a prospectively collected registry using the Utstein-style database. We obtained OHCA data from the database during the study period. Cardiac arrest was defined as the absence of signs of circulation, which was confirmed at the scene by EMTs. Patients with valid do-not-resuscitate (DNR) orders, obvious death at the scene without being transferred to hospital, aged younger than 18 years, and traumatic cardiac arrest (including hanging and drowning) were excluded from this study. The study was approved by the Institutional Review Board of the Ditmanson Medical Foundation Chia-Yi Christian Hospital (CYCH-IRB 2021016).

\subsection{EMS in Chiayi City}

Chiayi City is the second most densely populated city in Taiwan (4431.53 people per square kilometer), with an area of $60.02 \mathrm{~km}^{2}$ and 266,000 residents. Inhabitants over 65 years account for $16.2 \%$ of the population. There is one tertiary referral hospital, two secondary hospitals, and two primary hospitals in the city. The EMS system is based on the fire bureau and is composed of one centralized dispatch center and seven EMS stations. The EMS dispatch center functions $24-7$ and is operated by experienced EMTs. Once the dispatch center is called for medical assistance, the EMTs on duty are dispatched from the nearest EMT station. The protocol to determine patients with OHCA includes a few questions asked by the dispatcher on the phone; once the patients with OHCA are identified, dispatcher-assisted cardiopulmonary resuscitation (DACPR) is initiated simultaneously. For patients with OHCA, the basic life support (BLS) protocol used by all EMTs includes CPR, defibrillation (if feasible) by an automated external defibrillator (AED), and the use of bag-valve-mask or laryngeal mask airway. If EMT paramedics (EMT-P) are present at the scene, they can provide advanced life support (ALS), including epinephrine injection and endotracheal intubation, as appropriate. 
During the study period, all EMTs received regular training and performed CPR according to national guidelines based on the American Heart Association, European Resuscitation Council, and the International Liaison Committee on Resuscitation Guidelines $[17,18]$. The use of AEDs, maintenance of ventilation, and continued CPR during transportation to a hospital were mandatory for non-traumatic OHCA, unless ROSC was achieved. Quality control and assessment were conducted monthly to ensure resuscitation quality.

\subsection{Device and Implementation Timeline}

The Lund University Cardiac Assist System-2 (LUCAS-2) chest compression system (Physio-Control Inc., Redmond, WA, USA) is a portable mechanical piston CPR device. It is battery-driven and consists of an integrated suction cup to deliver automatic chest compression and active decompression back to the neutral position of the chest. It was designed to overcome the problems identified with manual chest compressions. The LUCAS-2 device assists rescuers by delivering effective, consistent and continuous chest compressions as recommended in the current resuscitation guidelines $[17,18]$. It is to be used for performing external cardiac compressions on adult cardiac arrest patients. It is indicated to use the LUCAS device unless it cannot be safely or correctly placed on the patient's chest or if the patient's body size is too small or too large to fit the device. In May 2018, Chiayi City EMS had initiated a 3-month pilot evaluation of LUCAS-2. Two devices were first provided at two of the seven EMS stations (one device at each EMS station). After device-specific training and evaluation, LUCAS-2 was formally deployed as standard equipment in September 2018, and since November 2019, all seven EMS stations have been equipped with the LUCAS-2. The implementation timeline of LUCAS-2 in the Chiayi EMS system is shown in Figure 1. The aim of training and assessment criteria for usage of LUCAS-2 is to be able to install the device and start CPR with less than $10 \mathrm{~s}$ of interruption at the scene. Once LUCAS-2 is applied, except for the pause to check the pulse and analyze the cardiac rhythm through AED, it will continuously perform CPR from the scene to the hospital. In order to ensure that the quality meets the criteria, the course of resuscitation in each mission is reviewed on a monthly basis through recorded videos by an internal review board consisting of an experienced EMT-P and a medical director. The application of LUCAS-2 is dependent on the dispatcher's identification of OHCA. In principle, however, LUCAS-2 is carried along with the dispatched EMTs unless OHCA has not been identified or the device is unavailable. When LUCAS- 2 is not used, manual CPR is performed. LUCAS-2 usage is marked on the EMS record sheet.

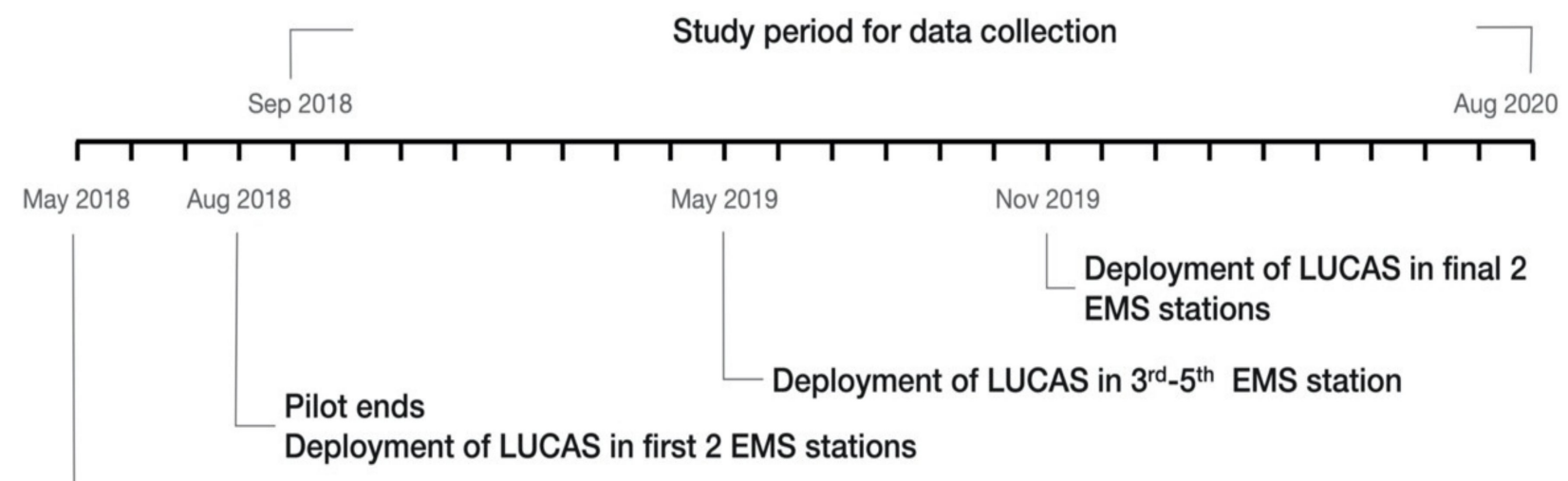

\section{Pilot begins in 2 EMS stations}

Figure 1. The implementation timeline of mechanical CPR device (LUCAS-2) in Chiayi EMS system. 


\subsection{Data Collection, Exposure, and Outcome}

We obtained data from the prospectively collected OHCA registry of Chiayi City. The collected data included the required information according to the Utstein-style guidelines, such as patient demographics, EMS response time (defined as the time from the ambulance leaving the EMS station to the rescue scene), EMS scene time (defined as the time from the ambulance arriving at the scene to leaving the scene), EMS transport time (defined as the time from the ambulance leaving the scene to arriving at the hospital), identification time of OHCA by dispatcher, start time of DACPR or bystander CPR, number of dispatched EMTs, characteristics of cardiac arrest (witness status, initial cardiac rhythm recording by AED), location of cardiac arrest (home, public area, medical institution (local clinic and nursing home), during ambulance transport, and others), pre-hospital treatment (including use of mechanical CPR, ventilation support by bag-valve-mask or laryngeal mask, intravenous fluid or epinephrine injection, total number of electric shocks by AED), the level of transferred hospital, achievement of ROSC at any time, a sustained ( $\geq 24 \mathrm{~h}$ ) ROSC, and survival at discharge with favorable neurologic status (GCS $\geq 13$ ).

The exposures in this study were defined as the use of mechanical CPR (LUCAS-2) during the pre-hospital stage and ambulance transport. The primary outcome was achievement of ROSC. The secondary outcomes were sustained ( $\geq 24 \mathrm{~h}$ ) ROSC and survival at discharge with favorable neurological status (GCS $\geq 13$ ).

\subsection{Statistical Analysis}

Based on the need to detect an odds ratio of 1.8, achieve ROSC using mechanical CPR, and set a ROSC rate of $20 \%$ at baseline using a two-sided test size of $5 \%$ and a power of $80 \%$, a total of 488 patients ( 244 in each group) would be required. Hence, we decided to include at least two years of data to meet this requirement.

Data of the included patients with OHCA were described and compared between the two groups, with and without the use of mechanical CPR. For continuous variables, Student's t-test (presented as mean \pm standard deviation) or Mann-Whitney $U$ test (presented as medians (interquartile range)) was used, as appropriate, according to the data distribution. For categorical variables (presented as number (percentage)), the chi-square test was used. To evaluate the net effect of mechanical CPR on patient outcomes, a forward stepwise logistic regression analysis was conducted, with adjustments for variables with $p$ value $<0.1$ as derived from the univariate analysis and the documented or possible predictors. The adjusted factors included age [19,20], sex [19], EMS response and scene time [21,22], number of dispatched EMTs, DACPR or bystander CPR [23-26], witnessed arrest, shockable rhythm [26], location of arrest [27], pre-hospital epinephrine injection [28,29], different batches of EMS stations with LUCAS-2 implementation, and level of transferred hospital [30]. Subgroup analyses with adjustment of the above-mentioned factors were also conducted to evaluate the net effect of mechanical CPR on different types of OHCA patients, including witness status, initial cardiac rhythm (shockable or non-shockable), different EMS response times ( $\leq$ and $>4 \mathrm{~min}$ ), and different age groups ( $<$ and $\geq 65$ years). Statistical significance was set at $p<0.05$. Statistical analysis was performed using the JASP Team (2020) JASP (Version 0.14.1) computer software.

\section{Results}

\subsection{Patient Population and Demographic Characteristics}

Between 1 September 2018 and 31 August 2020, 917 patients with OHCA who had activated the EMS system were identified. After excluding seven patients younger than 18 years of age, 286 patients either with DNR or not transferred to the hospital, and 72 patients with traumatic causes of cardiac arrest, a total of 552 patients with OHCA were included (Figure 2). Their median and mean age were $77(64-86)$ and $73.4( \pm 16.1)$ years, respectively. Among them, $72.6 \%$ were older than 65 years of age and $55.4 \%$ were male. Witnessed cardiac arrests had occurred in $50.9 \%$ of the patients and shockable rhythm in $23.7 \%$. The cardiac arrests occurred mostly at home (77\%). In total, 138 patients $(25 \%)$ had 
ROSC, 102 patients $(18.5 \%$ ) had sustained ( $\geq 24 \mathrm{~h})$ ROSC, and 27 patients $(4.9 \%)$ survived at discharge with favorable neurological status (GCS $\geq 13$ ).

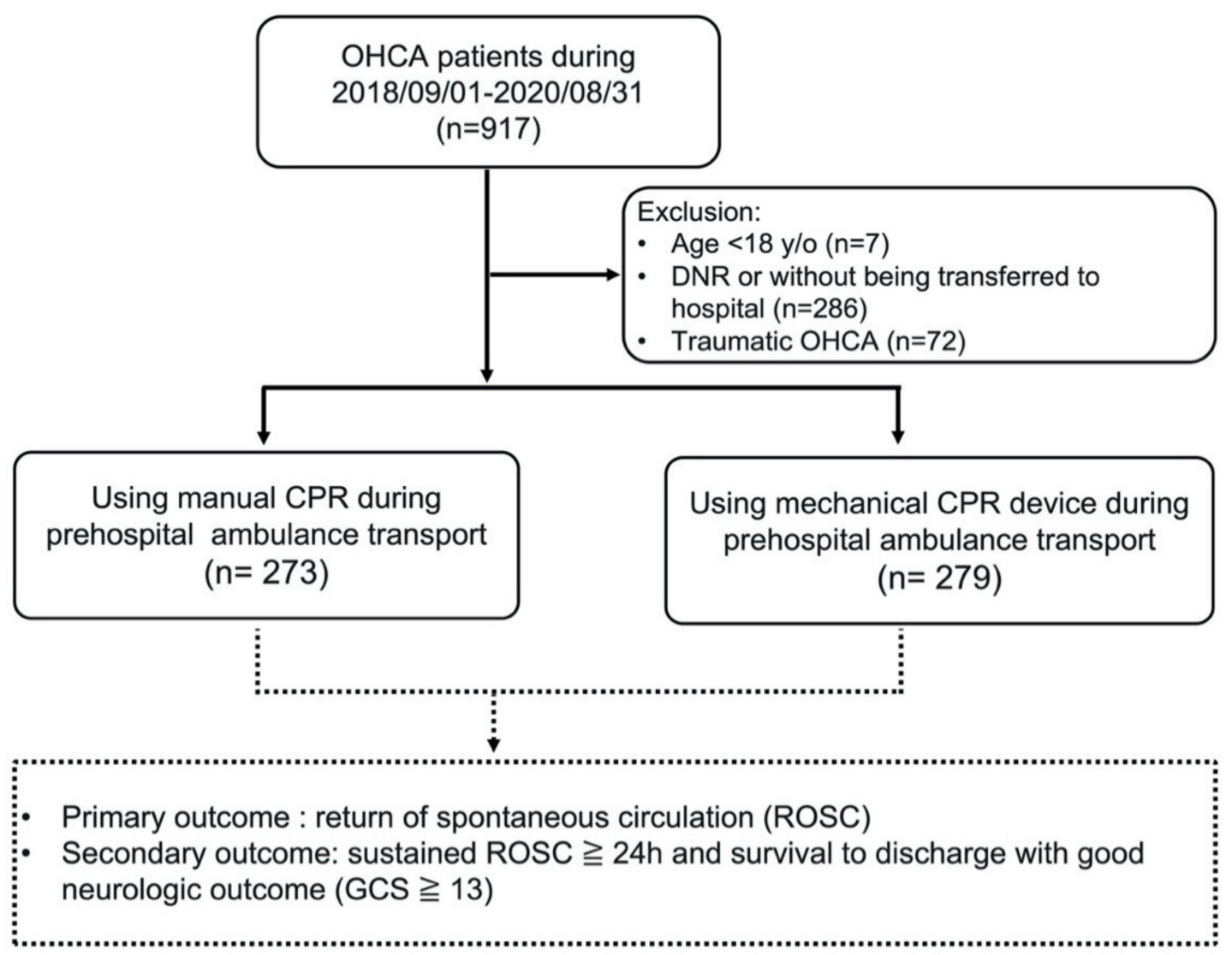

Figure 2. Flow chart of the patients included in the study.

Of the 552 patients, 279 (50.6\%) received mechanical CPR and 273 (49.4\%) received manual CPR at the pre-hospital stage (Figure 2). Table 1 shows the demographic characteristics of the patients treated with and without mechanical CPR. Compared to the manual CPR group, in the mechanical CPR group more patients received DACPR or bystander CPR $(p<0.001)$, the start time of DACPR was shorter $(p=0.03)$, more EMTs were dispatched $(p<0.001)$, the location of cardiac arrest was different $(p<0.001)$, the rate of administering mechanical CPR varied among the different batches of EMS stations that introduced LUCAS-2 $(p<0.001)$, and achievement of any ROSC $(p=0.044)$ and sustained $(\geq 24 \mathrm{~h}) \operatorname{ROSC}$ $(p=0.022)$ were higher. In addition, although not statistically significant, a shorter EMS response time $(p=0.075)$, longer EMS scene time $(p=0.061)$, and different proportion of transferred hospital levels $(p=0.079)$ were found in the mechanical CPR group than in the manual CPR group. There were no significant differences in age, sex, EMS transport time, identification time of OHCA by dispatcher, witnessed cardiac arrest, shockable rhythm, placement of laryngeal mask, intravenous fluid and epinephrine injection, total number of AED shocks, and the outcome of favorable neurologic status at discharge between the two groups (Table 1). 
Table 1. Comparison of clinical characteristics between manual and mechanical CPR.

\begin{tabular}{|c|c|c|c|c|c|}
\hline & \multicolumn{2}{|c|}{ Manual $(n=273)$} & \multicolumn{2}{|c|}{ Mechanical (LUCAS-2) $(n=279)$} & \multirow[t]{2}{*}{$p$ Value } \\
\hline \multicolumn{5}{|l|}{ Demographic characteristics } & \\
\hline Age & 77.5 & $(63-85)$ & 77.0 & $(65-86)$ & 0.619 \\
\hline Older adults ( $\geq 65$ years) & 193.0 & $(70.96)$ & 208.0 & (70.09) & 0.275 \\
\hline Male gender & 152 & $(55.68)$ & 154 & $(55.20)$ & 0.910 \\
\hline \multicolumn{6}{|l|}{ EMS time interval } \\
\hline Response time (min) & 4 & $(3-5)$ & 4 & $(2.5-5)$ & 0.075 \\
\hline Scene time (min) & 9 & $(7-12)$ & 10 & $(7-12)$ & 0.061 \\
\hline Transport time (min) & 3 & $(2-4)$ & 3 & $(2-4)$ & 0.194 \\
\hline Total EMS time (min) & 17 & $(14-20)$ & 17 & $(14.5-20)$ & 0.085 \\
\hline \multicolumn{6}{|l|}{ EMS Dispatcher } \\
\hline DACPR or BSCPR & 131 & $(47.99)$ & 197 & $(70.61)$ & $<0.001$ \\
\hline $\begin{array}{l}\text { Identification time of OHCA } \\
\text { by dispatcher (sec) }\end{array}$ & 63.5 & $(28-116.5)(n=168)$ & 58 & $(30-110)(n=238)$ & 0.380 \\
\hline Start time of DACPR (sec) & 199 & $(145-249)(n=115)$ & 167 & $(126-229)(n=177)$ & 0.030 \\
\hline $\begin{array}{l}\text { Number of dispatched EMT } \\
\text { (Mean } \pm \text { SD) }\end{array}$ & 2.78 & $(0.45)$ & 2.95 & $(0.33)$ & \multirow[t]{2}{*}{$<0.001$} \\
\hline $\begin{array}{l}\text { Number of dispatched EMT } \\
\text { (Median (IQR)) }\end{array}$ & 3 & $(3-3)$ & 3 & $(3-3)$ & \\
\hline \multicolumn{6}{|l|}{ Characteristics of arrest } \\
\hline Witnessed cardiac arrest & 146 & $(53.48)$ & 135 & $(48.39)$ & 0.231 \\
\hline $\begin{array}{l}\text { Shockable rhythm } \\
\text { (defibrillation) }\end{array}$ & 58 & $(21.25)$ & 73 & $(26.17)$ & 0.174 \\
\hline Location of arrest & & & & & \\
\hline Home & 198 & (72.53) & 227 & $(81.36)$ & \multirow{5}{*}{$<0.001$} \\
\hline Public area & 24 & $(8.79)$ & 17 & $(6.09)$ & \\
\hline Medical institution & 24 & $(8.79)$ & 34 & (12.19) & \\
\hline Others & 14 & (5.13) & 1 & $(0.36)$ & \\
\hline During ambulance transport & 13 & $(4.76)$ & 0 & $(0.00)$ & \\
\hline \multicolumn{6}{|l|}{ Pre-hospital treatment } \\
\hline Laryngeal mask airway & 218 & $(79.85)$ & 236 & $(84.59)$ & 0.146 \\
\hline Intravenous fluid injection & 10 & $(3.66)$ & 17 & $(6.09)$ & 0.186 \\
\hline Intravenous epinephrine & 10 & $(3.66)$ & 15 & $(5.38)$ & 0.333 \\
\hline Total number of AED shocks & 0 & $(0-0)$ & 0 & $(0-1)$ & 0.145 \\
\hline \multicolumn{6}{|l|}{$\begin{array}{l}\text { Different batches of EMS stations } \\
\text { with LUCAS-2 implementation }\end{array}$} \\
\hline The first batch (2 EMS stations) & 63 & $(23.08)$ & 110 & $(39.43)$ & \multirow{3}{*}{$<0.001$} \\
\hline $\begin{array}{l}\text { The second batch ( } 3 \text { EMS } \\
\text { stations) }\end{array}$ & 124 & $(45.42)$ & 114 & $(40.86)$ & \\
\hline $\begin{array}{l}\text { The final batch ( } 2 \text { EMS } \\
\text { stations) }\end{array}$ & 86 & $(31.50)$ & 55 & $(19.71)$ & \\
\hline \multicolumn{6}{|l|}{ Level of transferred hospital } \\
\hline Primary & 35 & $(12.82)$ & 48 & $(17.27)$ & \multirow{3}{*}{0.079} \\
\hline Secondary & 151 & $(55.31)$ & 128 & $(46.04)$ & \\
\hline Tertiary & 87 & $(31.87)$ & 102 & $(36.69)$ & \\
\hline \multicolumn{6}{|l|}{ Outcomes } \\
\hline Any ROSC & 58 & $(21.25)$ & 80 & $(28.67)$ & 0.044 \\
\hline Sustained $(\geq 24$ h) ROSC & 40 & $(14.65)$ & 62 & $(22.22)$ & 0.022 \\
\hline $\begin{array}{l}\text { Favorable neurologic status at } \\
\text { discharge (GCS } \geq 13 \text { ) }\end{array}$ & 12 & $(4.40)$ & 15 & $(5.38)$ & 0.593 \\
\hline
\end{tabular}

Values shown are $n(\%)$, mean $( \pm \mathrm{SD})$, or median (interquartile range). EMS: emergency medical services; EMT: emergency medical technician; CPR: cardiopulmonary resuscitation; DACPR: dispatcher-assisted CPR; BSCPR: bystander CPR; ROSC: return to spontaneous circulation; OHCA: out-of-hospital cardiac arrest; GCS: Glasgow Coma Scale. 


\subsection{Impacts of Mechanical CPR on Primary Outcome}

The net effect of mechanical CPR on the primary outcome-achievement of any ROSC was evaluated using multivariate analysis. We adjusted for the variables with $p$ values $<0.1$, derived from the univariate analysis (Table 1) and the documented or possible predictors associated with outcomes of OHCA, including age, sex, EMS response and scene time, number of dispatched EMTs, DACPR or bystander CPR, witnessed cardiac arrest, shockable rhythm, location of arrest, pre-hospital epinephrine injection, different batches of EMS stations with LUCAS-2 implementation, and level of transferred hospital (Table 2). The results showed that the use of mechanical CPR devices had significantly higher odds of achieving ROSC (odds ratio $(\mathrm{OR})=1.871,95 \%$ confidence interval $(\mathrm{CI}): 1.195-2.930, p=0.006)$. Moreover, every year of increasing age (OR $=0.979,95 \%$ CI: $0.966-0.992, p=0.001)$, witnessed cardiac arrest $(\mathrm{OR}=3.067,95 \% \mathrm{CI}: 1.966-4.786, p<0.001)$, cardiac arrest in a public area $(\mathrm{OR}=2.786,95 \% \mathrm{CI}$ : $1.319-5.886, p=0.007)$, and ambulance transport $(\mathrm{OR}=4.837,95 \%$ CI: $1.459-16.039, p=0.01)$ were independently associated with ROSC (Table 2). The final batch of EMS stations that introduced mechanical CPR showed a trend of lower odds of achievement of any ROSC than in the first batch of EMS stations ( $\mathrm{OR}=0.57,95 \% \mathrm{CI}: 0.318-1.020, p=0.058)$, but the difference was not statistically significant.

Table 2. Predictors associated with ROSC in OHCA patients.

\begin{tabular}{|c|c|c|c|c|c|c|}
\hline Parameters & OR & $(95 \% \mathrm{CI})$ & $p$ Value & aOR & $(95 \% \mathrm{CI})$ & $p$ Value \\
\hline Age (per year) & 0.978 & $(0.967-0.990)$ & $<0.001$ & 0.979 & $(0.966-0.992)$ & 0.001 \\
\hline Male gender & 1.103 & $(0.748-1.627)$ & 0.621 & & - & \\
\hline EMS response time (per minute) & 0.912 & $(0.822-1.013)$ & 0.085 & & - & \\
\hline EMS scene time (per minute) & 0.952 & $(0.909-0.998)$ & 0.04 & & - & \\
\hline Number of dispatched EMT & 1.259 & $(0.770-2.060)$ & 0.359 & & - & \\
\hline DACPR or BSCPR & 0.758 & $(0.513-1.118)$ & 0.162 & & - & \\
\hline Witnessed cardiac arrest & 2.957 & $(1.956-4.471)$ & $<0.001$ & 3.067 & $(1.966-4.786)$ & $<0.001$ \\
\hline Shockable rhythm & 1.598 & $(1.037-2.460)$ & 0.033 & & - & \\
\hline \multicolumn{7}{|l|}{ Location of arrest } \\
\hline Home & reference & & & reference & & \\
\hline Public area & 4.25 & $(2.205-8.191)$ & $<0.001$ & 2.786 & $(1.319-5.886)$ & 0.007 \\
\hline Medical institution & 0.957 & $(0.487-1.883)$ & 0.9 & 0.989 & $(0.487-2.007)$ & 0.976 \\
\hline Others & 1.835 & $(0.612-5.503)$ & 0.279 & 1.805 & $(0.556-5.866)$ & 0.326 \\
\hline During ambulance transport & 5.873 & $(1.876-18.383)$ & 0.002 & 4.837 & $(1.459-16.039)$ & 0.01 \\
\hline Pre-hospital epinephrine injection & 1.735 & $(0.749-4.022)$ & 0.199 & & - & \\
\hline \multicolumn{7}{|c|}{ Different batches of EMS stations with LUCAS-2 implementation } \\
\hline The first batch (2 EMS stations) & reference & & & reference & & \\
\hline The second batch (3 EMS stations) & 0.694 & $(0.448-1.076)$ & 0.102 & 0.794 & $(0.492-1.281)$ & 0.345 \\
\hline The final batch (2 EMS stations) & 0.522 & $(0.308-0.885)$ & 0.016 & 0.57 & $(0.318-1.020)$ & 0.058 \\
\hline \multicolumn{7}{|l|}{ Level of transferred hospital } \\
\hline Primary & reference & & & & - & \\
\hline Secondary & 0.962 & $(0.536-1.727)$ & 0.898 & & - & \\
\hline Tertiary & 1.455 & $(0.799-2.647)$ & 0.22 & & - & \\
\hline Mechanical CPR & 1.49 & (1.010-2.199) & 0.045 & 1.871 & (1.195-2.930) & 0.006 \\
\hline
\end{tabular}

OR: odds ratio; aOR: adjusted odds ratio; CI: confidence interval; EMS: emergency medical services; EMT: emergency medical technician; CPR: cardiopulmonary resuscitation; DACPR: dispatcher-assisted CPR; BSCPR: bystander CPR; ROSC: return to spontaneous circulation; OHCA: out-of-hospital cardiac arrest. 


\subsection{Impacts of Mechanical CPR on Secondary Outcomes}

The net effect of mechanical CPR on secondary outcomes, i.e., achievement of sustained ( $\geq 24 \mathrm{~h}$ ) ROSC (Table 3 ) and survival at discharge with favorable neurologic status (GCS $\geq 13$ ) (Table 4), was evaluated by multivariate analysis after adjusting for influencing factors. The use of mechanical CPR devices showed a significant association with achievement of sustained ( $\geq 24 \mathrm{~h}$ ) ROSC (OR $=2.353,95 \%$ CI: 1.427-3.879, $p<0.001$ ) (Table 3). Every year of increasing age (OR $=0.984,95 \%$ CI: $0.970-0.998, p=0.023)$, every minute of increasing EMS scene time (OR $=0.939,95 \%$ CI: 0.887-0.994, $p=0.029)$, witnessed cardiac arrest $(\mathrm{OR}=2.069,95 \% \mathrm{CI}: 1.276-3.352, p=0.003)$, cardiac arrest in public areas $(\mathrm{OR}=3.187$, $95 \%$ CI: $1.492-6.808, p=0.003$ ), and ambulance transport (OR $=5.527,95 \%$ CI: $1.666-18.333$, $p=0.005)$ were independently associated with sustained ( $\geq 24 \mathrm{~h}$ ) ROSC (Table 2$)$. However, the use of mechanical CPR devices did not show a significant association with survival at discharge with favorable neurological status (GCS $\geq 13)(\mathrm{OR}=1.066,95 \% \mathrm{CI}: 0.459-2.475$, $p=0.881$ ) (Table 4). The independent factors associated with this outcome were every year of increasing age ( $\mathrm{OR}=0.967,95 \% \mathrm{CI}: 0.945-0.989, p=0.004)$, witnessed cardiac arrest $(\mathrm{OR}=4.016,95 \% \mathrm{CI}: 1.455-11.08, p=0.007)$, and shockable rhythm $(\mathrm{OR}=6.881,95 \% \mathrm{CI}$ : 2.844-16.653, $p<0.001$ ) (Table 4).

Table 3. Predictors associated with sustained ROSC $\geq 24 \mathrm{~h}$ in OHCA patients.

\begin{tabular}{|c|c|c|c|c|c|c|}
\hline Parameters & OR & $(95 \% \mathrm{CI})$ & $p$ Value & aOR & $(95 \% \mathrm{CI})$ & $p$ Value \\
\hline Age (per year) & 0.982 & $(0.969-0.994)$ & 0.004 & 0.984 & $(0.970-0.998)$ & 0.023 \\
\hline Male gender & 0.884 & $(0.574-1.360)$ & 0.575 & & - & \\
\hline EMS response time (per minute) & 0.853 & $(0.753-0.965)$ & 0.012 & & - & \\
\hline EMS scene time (per minute) & 0.928 & $(0.877-0.981)$ & 0.008 & 0.939 & $(0.887-0.994)$ & 0.029 \\
\hline Number of dispatched EMT & 1.463 & $(0.833-2.568)$ & 0.185 & & - & \\
\hline DACPR or BSCPR & 1.128 & $(0.725-1.753)$ & 0.593 & & - & \\
\hline Witnessed cardiac arrest & 2.347 & $(1.490-3.697)$ & $<0.001$ & 2.069 & $(1.276-3.352)$ & 0.003 \\
\hline Shockable rhythm & 1.82 & $(1.138-2.908)$ & 0.012 & & - & \\
\hline \multicolumn{7}{|l|}{ Location of arrest } \\
\hline Home & reference & & & reference & & \\
\hline Public area & 4.497 & $(2.296-8.808)$ & $<0.001$ & 3.187 & $(1.492-6.808)$ & 0.003 \\
\hline Medical institution & 1.345 & $(0.662-2.732)$ & 0.413 & 1.33 & $(0.640-2.764)$ & 0.445 \\
\hline Others & 2.089 & $(0.645-6.768)$ & 0.219 & 2.795 & $(0.785-9.959)$ & 0.113 \\
\hline During ambulance transport & 4.925 & (1.603-15.137) & 0.005 & 5.527 & $(1.666-18.333)$ & 0.005 \\
\hline Pre-hospital epinephrine injection & 1.418 & $(0.552-3.644)$ & 0.469 & & - & \\
\hline \multicolumn{7}{|c|}{ EMS stations with different batches of LUCAS-2 implementation } \\
\hline The first batch (2 EMS stations) & reference & & & & - & \\
\hline The second batch ( 3 EMS stations) & 0.692 & $(0.425-1.127)$ & 0.139 & & - & \\
\hline The final batch (2 EMS stations) & 0.582 & $(0.325-1.042)$ & 0.069 & & - & \\
\hline \multicolumn{7}{|l|}{ Level of transferred hospital } \\
\hline Primary & reference & & & & - & \\
\hline Secondary & 0.848 & $(0.452-1.591)$ & 0.608 & & - & \\
\hline Tertiary & 1.089 & $(0.569-2.084)$ & 0.797 & & - & \\
\hline Mechanical CPR & 1.664 & (1.074-2.580) & 0.023 & 2.353 & (1.427-3.879) & $<0.001$ \\
\hline
\end{tabular}

OR: odds ratio; aOR: adjusted odds ratio; CI: confidence interval; EMS: emergency medical services; EMT: emergency medical technician; CPR: cardiopulmonary resuscitation; DACPR: dispatcher-assisted CPR; BSCPR: bystander CPR; ROSC: return to spontaneous circulation; OHCA: out-of-hospital cardiac arrest. 
Table 4. Predictors associated with favorable neurologic status at discharge (GCS $\geq 13$ ) in OHCA patients.

\begin{tabular}{|c|c|c|c|c|c|c|}
\hline Parameters & OR & $(95 \% \mathrm{CI})$ & $p$ Value & $\mathrm{aOR}$ * & $(95 \% \mathrm{CI})$ & $p$ Value \\
\hline Age (per year) & 0.964 & $(0.944-0.984)$ & $<0.001$ & 0.967 & $(0.945-0.989)$ & 0.004 \\
\hline Male gender & 1.388 & $(0.624-3.089)$ & 0.421 & & - & \\
\hline EMS response time (per minute) & 0.963 & $(0.787-1.180)$ & 0.719 & & - & \\
\hline EMS scene time (per minute) & 0.967 & $(0.881-1.060)$ & 0.471 & & - & \\
\hline Number of dispatched EMT & 1.536 & $(0.554-4.257)$ & 0.41 & & - & \\
\hline DACPR or BSCPR & 1.17 & $(0.526-2.604)$ & 0.701 & & - & \\
\hline Witnessed cardiac arrest & 4.519 & $(1.686-12.112)$ & 0.003 & 4.016 & $(1.455-11.080)$ & 0.007 \\
\hline Shockable rhythm & 8.758 & $(3.736-20.531)$ & $<0.001$ & 6.881 & $(2.844-16.653)$ & $<0.001$ \\
\hline \multicolumn{7}{|l|}{ Location of arrest } \\
\hline Home & reference & & & & & \\
\hline Public area & 4.655 & $(1.817-11.924)$ & 0.001 & & - & \\
\hline Medical institution & 0.397 & $(0.052-3.028)$ & 0.373 & & - & \\
\hline Others & & $(\mathrm{N} / \mathrm{A})$ & 0.989 & & - & \\
\hline During ambulance transport & 1.884 & $(0.232-15.294)$ & 0.553 & & - & \\
\hline Pre-hospital epinephrine injection & 1.746 & $(0.390-7.823)$ & 0.466 & & - & \\
\hline \multicolumn{7}{|c|}{ EMS stations with different batches of LUCAS-2 implementation } \\
\hline The first batch (2 EMS stations) & reference & & & & - & \\
\hline The second batch ( 3 EMS stations) & 0.968 & $(0.398-2.350)$ & 0.942 & & - & \\
\hline The final batch (2 EMS stations) & 0.81 & & 0.696 & & - & \\
\hline Level of transferred hospital & & $(0.281-2.332)$ & & & - & \\
\hline Primary & reference & & & & - & \\
\hline Secondary & 2.14 & $(0.476-9.612)$ & 0.321 & & - & \\
\hline Tertiary & 2.503 & $(0.542-11.551)$ & 0.24 & & - & \\
\hline Mechanical CPR & 1.236 & $(0.568-2.691)$ & 0.594 & 1.066 & $(0.459-2.475)$ & 0.881 \\
\hline
\end{tabular}

OR: odds ratio; aOR: adjusted odds ratio; CI: confidence interval; EMS: emergency medical services; EMT: emergency medical technician; CPR: cardiopulmonary resuscitation; DACPR: dispatcher-assisted CPR; BSCPR: bystander CPR; ROSC: return to spontaneous circulation; OHCA: out-of-hospital cardiac arrest. * Adjusting factors include age, witnessed cardiac arrest, and shockable rhythm.

\subsection{Subgroup Analysis of the Effect of Mechanical CPR on Different Status of OHCA Patients}

To evaluate the net effect of mechanical CPR on the various status of OHCA patients, we performed subgroup analyses based on witness status, initial AED cardiac rhythm, different EMS response times ( $\leq 4 \mathrm{~min}$ and $>4 \mathrm{~min}$ ), and different age groups ( $<65$ years and $\geq 65$ years) (Figure 3 ). The possible influencing factors mentioned above were also adjusted. The results showed that mechanical CPR was independently associated with achievement of any ROSC (aOR $=2.693,95 \%$ CI: $1.512-4.796, p=0.001)$ and sustained $(\geq 24 \mathrm{~h})$ ROSC $(\mathrm{aOR}=2.524,95 \%$ CI: 1.318-4.831, $p=0.005)$ in witnessed OHCA patients but not in non-witnessed OHCA patients. Regarding the initial AED rhythm, mechanical CPR was independently associated with any ROSC (aOR $=1.919,95 \%$ CI: 1.148-3.209, $p=0.013)$ and sustained $(\geq 24 \mathrm{~h}) \mathrm{ROSC}(\mathrm{aOR}=2.63,95 \% \mathrm{CI}: 1.428-4.842, p=0.002)$ in OHCA patients with non-shockable rhythm but not in those with shockable rhythm. For different EMS response times, mechanical CPR showed a significant association with any $\mathrm{ROSC}(\mathrm{aOR}=2.514,95 \%$ CI: $1.411-4.481, p=0.002)$ and sustained $(\geq 24 \mathrm{~h}$ ) ROSC (aOR $=2.725,95 \% \mathrm{CI}: 1.455-5.105$, $p=0.002)$ in OHCA patients with shorter EMS response time $(\leq 4 \mathrm{~min})$ but not in those with longer EMS response time ( $>4 \mathrm{~min}$ ). Moreover, mechanical CPR was significantly associated with any ROSC ( $\mathrm{aOR}=1.75,95 \% \mathrm{CI}$ : 1.022-2.999, $p=0.042$ ) and sustained ( $\geq 24 \mathrm{~h}$ ) ROSC (aOR $=1.796,95 \%$ CI: $1.022-3.154, p=0.042)$ in patients aged $\geq 65$ years and with any $\operatorname{ROSC}(\mathrm{aOR}=2.666,95 \% \mathrm{CI}: 1.213-5.859, p=0.015)$ in patients aged $<65$ years. 


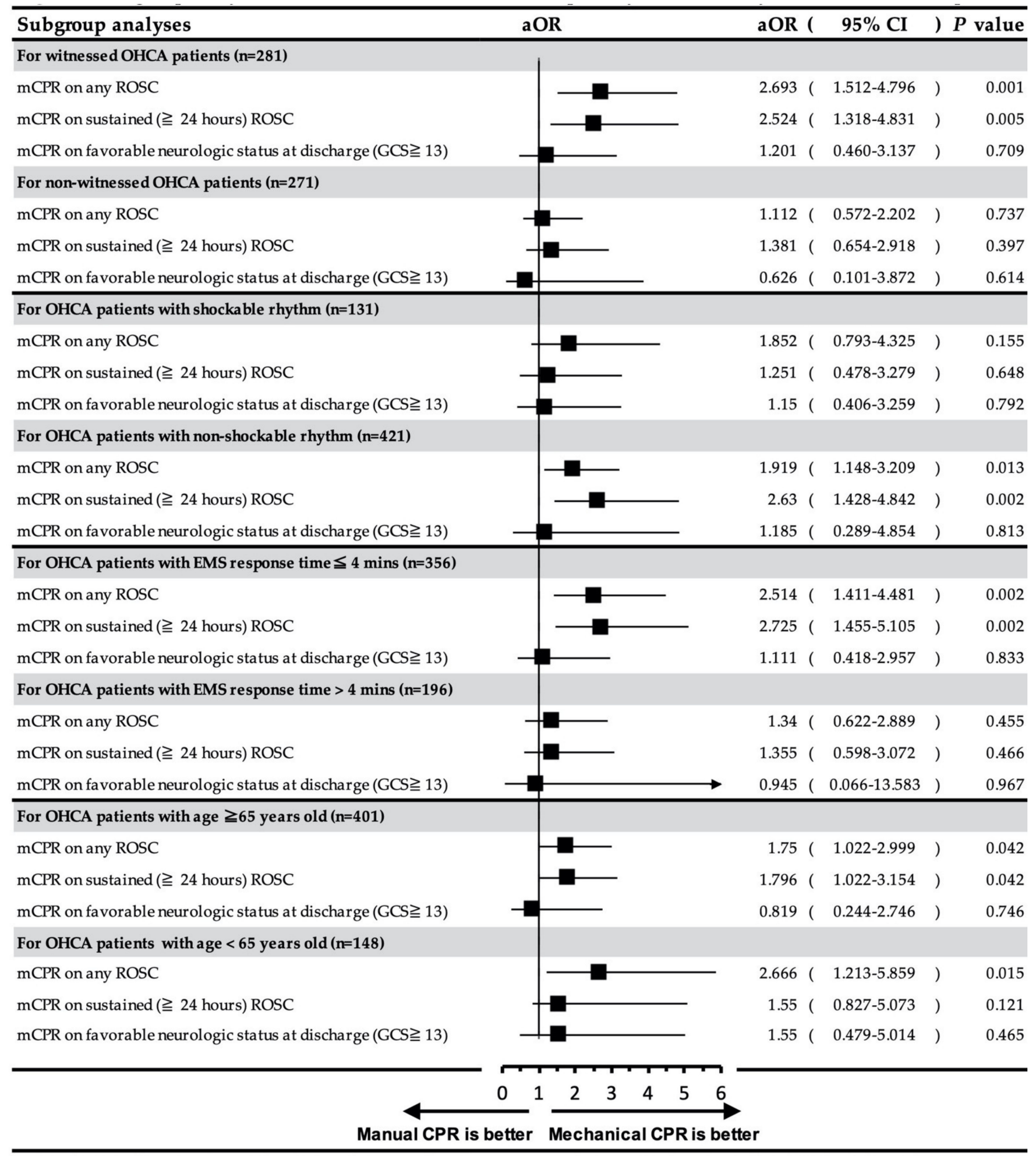

Figure 3. Subgroup analyses of mechanical CPR devices on the primary and secondary outcomes of OHCA patients. aOR: Adjusted odds ratio; CI: confidence interval; CPR: cardiopulmonary resuscitation; mCPR: mechanical CPR; DACPR: dispatcher-assisted CPR; BSCPR: bystander CPR; ROSC: return to spontaneous circulation; OHCA: out-of-hospital cardiac arrest. 


\section{Discussion}

Our findings showed that, in OHCA patients, the use of mechanical CPR devices at the scene and during ambulance transport was associated with the achievement of any ROSC and sustained ( $\geq 24 \mathrm{~h}$ ) ROSC, after adjustment for the independent influencing factors of ROSC (Tables 2 and 3). During subgroup analyses, we further found that mechanical CPR devices were more effective than manual CPR in achieving ROSC, especially in patients with witnessed cardiac arrest, non-shockable rhythm, and short EMS response time (Figure 3). These findings support the importance of early EMS activation and early high-quality CPR at the pre-hospital stage.

Our study revealed that the pre-hospital use of mechanical CPR did not have any benefits regarding survival at discharge with favorable neurologic status. The independent factors most associated with this outcome were younger age, witnessed cardiac arrest, and shockable rhythm (Table 4). This finding may be reasonable because many factors can influence the outcome, such as the patient's underlying condition, etiologies of cardiac arrest, level of the receiving hospital, in-hospital post-cardiac arrest care, and post-resuscitation care. Without appropriate control of these influencing factors, the effects of prehospital use of mechanical CPR devices may not be evident. Hence, although long-term survival with favorable neurological status would be a more relevant outcome in assessing the quality of resuscitation in OHCA patients, we set ROSC, which is a more reliable, wellrecognized, and easily obtainable outcome, as the primary outcome to evaluate the effect of implementing mechanical CPR devices in our EMS system.

The resuscitative effects of mechanical CPR devices in patients with OHCA remain controversial. A previous meta-analysis pooled the studies of both the "in-hospital" and "pre-hospital" use of mechanical CPR devices and found that there was no survival benefit in using mechanical CPR devices $[13,31]$. However, the medical resources at in-hospital settings can be expected to be better than those in the pre-hospital setting, such as a more spacious environment, more staff for maintaining good chest compression, and more medication and equipment for advanced cardiac life support. Hence, it is reasonable that almost all studies evaluating the "in-hospital" use of mechanical CPR for OHCA patients did not show survival benefits in comparison with manual CPR [5-7,32]. Moreover, although large randomized controlled trials in pre-hospital settings have not shown the benefits of the routine use of mechanical CPR devices [8-11], our study results as well as the majority of the observational studies suggest an advantage over manual CPR for ROSC and survival to hospital admission $[12,13,33]$. When we compared the main difference between our study and previous controlled trials [9-11], we found an obviously shorter EMS response time (median time: 4 vs. 6-10 min), shorter total EMS time (median time: 17 vs. $36-47 \mathrm{~min}$ ), and a higher proportion of patients receiving placement of advanced airway ( $82 \%$ vs. $26 \%$ ) in our study (Table 1$)$. Moreover, in our subgroup analysis, we found that the survival benefits of mechanical CPR devices were evident in patients after a shorter EMS response time ( $\leq 4 \mathrm{~min}$ ) than after a longer EMS response time ( $>4 \mathrm{~min}$ ) (Figure 3 ). It can therefore be assumed that the benefits of mechanical CPR emerge only when the device is applied early on-site. Further studies are required to evaluate the effect of early applied mechanical CPR devices.

In addition, our study found that the use of mechanical CPR devices was associated with ROSC, especially in witnessed cardiac arrests (Figure 3). Early high-quality CPR is important to patient outcome. In patients with non-witnessed cardiac arrests, a longer noflow interval (duration from cardiac arrest to the start of CPR) can be expected. Therefore, even if mechanical CPR is applied to these patients, its effects on patient outcomes maybe inadequate. Furthermore, our study found that the use of mechanical CPR was more significantly associated with ROSC in patients with non-shockable rhythm than in those with shockable rhythm (Figure 3). This may be reasonable because, in OHCA patients with non-shockable rhythm, early high-quality CPR may be the most important pre-hospital factor for ROSC. In OHCA patients with shockable rhythm, in addition to high-quality CPR, early defibrillation is crucial for ROSC [4]. The relatively low number of patients 
with shockable rhythm $(n=131)$ in our study may have influenced the statistical power. However, although not statistically significant, we did find that the use of mechanical CPR devices in OHCA patients with shockable rhythm resulted in higher odds of achieving ROSC (Figure 3). Age is an independent factor that influences the outcomes of patients with OHCA $[19,20]$. In our study, every year of increasing age showed significantly lower odds of achievement of any ROSC, sustained ROSC, and survival at discharge with favorable neurologic status (Tables 2-4). A recent study in Taiwan demonstrated that pre-hospital prognostic factors in OHCA patients varied in different age groups [34]. However, in our study, we found that the use of mechanical CPR devices benefited the achievement of ROSC both in younger ( $<65$ years) and older patients with OHCA ( $\geq 65$ years) (Figure 3 ).

This observational study had several limitations. First, we were not able to obtain the background of the patients and their pre-existing conditions as they were sent to different hospitals. These underlying factors may influence patient outcomes. However, this occurs in most OHCA studies. Further study may be needed to control the influence of a patient's underlying disease. Second, as with other OHCA registry analyses, we could not obtain and analyze the management that patients received at the in-hospital care stage. The post-cardiac arrest care, like targeted temperature management and coronary angiography with reperfusion therapy, are associated with a patient's neurological outcomes. Without adjusting for these factors, the impact of pre-hospital use of mechanical CPR devices may not be evident. Instead, in order to control the factors in the in-hospital stage, we adjusted for the hospital factors based on the level of the hospital where the patients were transferred in this study. This is also the reason that we did not set the outcome of survival with favorable neurological status at discharge as our primary outcome. Third, the time of implementing LUCAS-2 in different EMS stations in our city was different; hence, the EMTs' familiarity with the mechanical CPR device may affect the patient's outcome. However, we adjusted for the factor of EMS stations with different times of LUCAS-2 implementation in the multivariate analysis. Finally, since this is a retrospective study, the patient's outcome was collected from different hospital systems. There was no unified scoring system, like Glasgow-Pittsburgh cerebral performance categories, to record a patient's neurologic status at discharge; we only could obtain the most simplified score, GCS, as the measured outcome in patient's neurological status at discharge.

\section{Conclusions}

After a two-year period of implementing mechanical CPR devices in the EMS system, the pre-hospital use of mechanical CPR devices was significantly associated with an increased rate of ROSC in patients with OHCA. We further found that the survival benefits of mechanical CPR devices were evident in patients with a shorter $(\leq 4 \mathrm{~min})$ EMS response time and patients with witnessed cardiac arrest. These findings suggest that the benefits of mechanical CPR emerge only when the device is applied early on-site and echo that survival in cardiac arrest is highly dependent on early recognition and early application of high-quality CPR. This also may be the reason why previous controlled trials failed to show the advantages of mechanical CPR devices, because compared with this study, there was a time delay in application of the mechanical CPR devices. Hence, it is necessary to conduct further studies to evaluate the effect of early applied mechanical CPR devices on patients with witnessed cardiac arrests after appropriate adjustment of the patients' underlying diseases and the management of post-resuscitation care.

Supplementary Materials: The following is available online at https:/ /www.mdpi.com/article/10.339 0/ijerph18073636/s1, Supplementary Table S1: Summary of the previous studies investigating prehospital use of mechanical CPR devices on the outcome of patients with OHCA.

Author Contributions: M.-J.T. and C.-F.H. conceived the study and developed the study protocols. Y.-R.C., H.-C.H., C.-H.T., Y.-S.S., C.-H.L. and C.-F.H. provided technical and administrative support and contributed to the acquisition of data. M.-J.T. performed statistical analysis. Y.-R.C., C.-J.L., C.-F.H. and M.-J.T. interpreted the study results and drafted the manuscript. C.-F.H. and M.-J.T. are 
the corresponding authors who take responsibility for this paper. All authors have read and agreed to the published version of the manuscript.

Funding: This research was funded by Ditmanson Medical Foundation Chia-Yi Christian Hospital Research Program, grant number R110-015.

Institutional Review Board Statement: The study was conducted according to the guidelines of the Declaration of Helsinki and approved by the Institutional Review Board of the Ditmanson Medical Foundation Chia-Yi Christian Hospital (CYCH-IRB 2021016).

Informed Consent Statement: The Institutional Review Board of the Ditmanson Medical Foundation Chia-Yi Christian Hospital (CYCH-IRB 2021016) has approved this study to be exempt from informed consent.

Data Availability Statement: No new data were created or analyzed in this study.

Acknowledgments: We appreciate the dedication of the Chiayi City fire bureau.

Conflicts of Interest: The authors declare no conflict of interest.

\section{References}

1. Kudenchuk, P.J.P.; Sandroni, C.C.; Drinhaus, H.H.; Böttiger, B.W.; Cariou, A.A.; Sunde, K.K.; Dworschak, M.M.; Taccone, F.S.; Deye, N.N.; Friberg, H.; et al. Breakthrough in cardiac arrest: Reports from the 4th Paris International Conference. Ann. Intensive Care 2015, 5, 22. [CrossRef] [PubMed]

2. Berdowski, J.; Berg, R.A.; Tijssen, J.G.; Koster, R.W. Global incidences of out-of-hospital cardiac arrest and survival rates: Systematic review of 67 prospective studies. Resuscitation 2010, 81, 1479-1487. [CrossRef] [PubMed]

3. Wang, C.-Y.; Wang, J.-Y.; Teng, N.-C.; Chao, T.-T.; Tsai, S.-L.; Chen, C.-L.; Hsu, J.-Y.; Wu, C.-P.; Lai, C.-C.; Chen, L. The Secular Trends in the Incidence Rate and Outcomes of Out-of-Hospital Cardiac Arrest in Taiwan-A Nationwide Population-Based Study. PLoS ONE 2015, 10, e0122675. [CrossRef] [PubMed]

4. Merchant, R.M.; Topjian, A.A.; Panchal, A.R.; Cheng, A.; Aziz, K.; Berg, K.M.; Lavonas, E.J.; Magid, D.J. Part 1: Executive Summary: 2020 American Heart Association Guidelines for Cardiopulmonary Resuscitation and Emergency Cardiovascular Care. Circulation 2020, 142, S337-S357. [CrossRef]

5. Kim, H.T.; Kim, J.G.; Jang, Y.S.; Kang, G.H.; Kim, W.; Choi, H.Y.; Jun, G.S. Comparison of in-hospital use of mechanical chest compression devices for out-of-hospital cardiac arrest patients: AUTOPULSE vs. LUCAS. Medicine 2019, 98, e17881. [CrossRef]

6. Hayashida, K.; Tagami, T.; Fukuda, T.; Suzuki, M.; Yonemoto, N.; Kondo, Y.; Ogasawara, T.; Sakurai, A.; Tahara, Y.; Nagao, K.; et al. Mechanical Cardiopulmonary Resuscitation and Hospital Survival Among Adult Patients with Nontraumatic Out-of-Hospital Cardiac Arrest Attending the Emergency Department: A Prospective, Multicenter, Observational Study in Japan (SOS-KANTO [Survey of Survivors after Out-of-Hospital Cardiac Arrest in Kanto Area] 2012 Study). J. Am. Heart Assoc. 2017, 6, 007420. [CrossRef]

7. Lin, C.-K.; Huang, M.-C.; Feng, Y.-T.; Jeng, W.-H.; Chung, T.-C.; Lau, Y.-W.; Te-Cheng, C. Effectiveness of mechanical chest compression for out-of-hospital cardiac arrest patients in an emergency department. J. Chin. Med. Assoc. 2015, 78, 360-363. [CrossRef]

8. Gates, S.; Lall, R.; Quinn, T.; Deakin, C.D.; Cooke, M.W.; Horton, J.; Lall, R.; Lamb, S.E.; McCabe, C.; Quinn, T.; et al. Prehospital randomised assessment of a mechanical compression device in out-of-hospital cardiac arrest (PARAMEDIC): A pragmatic, cluster randomised trial and economic evaluation. Health Technol. Assess 2017, 21. [CrossRef]

9. Perkins, G.D.; Lall, R.; Quinn, T.; Deakin, C.D.; Cooke, M.W.; Horton, J.; Lamb, S.E.; Slowther, A.-M.; Woollard, M.; Carson, A.; et al. Mechanical versus manual chest compression for out-of-hospital cardiac arrest (PARAMEDIC): A pragmatic, cluster randomised controlled trial. Lancet 2015, 385, 947-955. [CrossRef]

10. Wik, L.; Olsen, J.-A.; Persse, D.; Sterz, F.; Lozano, M.; Brouwer, M.A.; Westfall, M.; Souders, C.M.; Malzer, R.; van Grunsven, P.M.; et al. Manual vs. integrated automatic load-distributing band CPR with equal survival after out of hospital cardiac arrest. The randomized CIRC trial. Resuscitation 2014, 85, 741-748. [CrossRef]

11. Rubertsson, S.; Lindgren, E.; Smekal, D.; Östlund, O.; Silfverstolpe, J.; Lichtveld, R.A.; Boomars, R.; Ahlstedt, B.; Skoog, G.; Kastberg, R.; et al. Mechanical Chest Compressions and Simultaneous Defibrillation vs Conventional Cardiopulmonary Resuscitation in Out-of-Hospital Cardiac Arrest: The LINC randomized trial. JAMA 2014, 311, 53-61. [CrossRef]

12. Seewald, S.; Obermaier, M.; Lefering, R.; Bohn, A.; Georgieff, M.; Muth, C.-M.; Gräsner, J.-T.; Masterson, S.; Scholz, J.; Wnent, J. Application of mechanical cardiopulmonary resuscitation devices and their value in out-of-hospital cardiac arrest: A retrospective analysis of the German Resuscitation Registry. PLOS ONE 2019, 14, e0208113. [CrossRef]

13. Bonnes, J.L.; Brouwer, M.A.; Navarese, E.P.; Verhaert, D.V.; Verheugt, F.W.; Smeets, J.L.; De Boer, M.-J. Manual Cardiopulmonary Resuscitation Versus CPR Including a Mechanical Chest Compression Device in Out-of-Hospital Cardiac Arrest: A Comprehensive Meta-Analysis from Randomized and Observational Studies. Ann. Emerg. Med. 2016, 67, 349-360.e3. [CrossRef]

14. Gyory, R.A.; Buchle, S.E.; Rodgers, D.; Lubin, J.S. The Efficacy of LUCAS in Prehospital Cardiac Arrest Scenarios: A Crossover Mannequin Study. West. J. Emerg. Med. 2017, 18, 437-445. [CrossRef] 
15. Fox, J.; Fiechter, R.; Gerstl, P.; Url, A.; Wagner, H.; Lüscher, T.F.; Eriksson, U.; Wyss, C.A. Mechanical versus manual chest compression CPR under ground ambulance transport conditions. Acute Card. Care 2013, 15, 1-6. [CrossRef]

16. Kahn, P.A.; Dhruva, S.S.; Rhee, T.G.; Ross, J.S. Use of Mechanical Cardiopulmonary Resuscitation Devices for Out-of-Hospital Cardiac Arrest, 2010-2016. JAMA Netw. Open 2019, 2, e1913298. [CrossRef]

17. Kleinman, M.E.; Brennan, E.E.; Goldberger, Z.D.; Swor, R.A.; Terry, M.; Bobrow, B.J.; Gazmuri, R.J.; Travers, A.H.; Rea, T.D. Part 5: Adult Basic Life Support and Cardiopulmonary Resuscitation Quality: 2015 American Heart Association Guidelines Update for Cardiopulmonary Resuscitation and Emergency Cardiovascular Care. Circulation 2015, 132, S414-S435. [CrossRef]

18. Travers, A.H.; Rea, T.D.; Bobrow, B.J.; Edelson, D.P.; Berg, R.A.; Sayre, M.R.; Berg, M.D.; Chameides, L.; O'Connor, R.E.; Swor, R.A. Part 4: CPR Overview: 2010 American Heart Association Guidelines for Cardiopulmonary Resuscitation and Emergency Cardiovascular Care. Circulation 2010, 122, S676-S684. [CrossRef]

19. Hagihara, A.; Onozuka, D.; Ono, J.; Nagata, T.; Hasegawa, M. Age $\times$ Gender Interaction Effect on Resuscitation Outcomes in Patients with Out-of-Hospital Cardiac Arrest. Am. J. Cardiol. 2017, 120, 387-392. [CrossRef]

20. Andersen, L.W.; Bivens, M.J.; Giberson, T.; Giberson, B.; Mottley, J.L.; Gautam, S.; Salciccioli, J.D.; Cocchi, M.N.; McNally, B.; Donnino, M.W. The relationship between age and outcome in out-of-hospital cardiac arrest patients. Resuscitation 2015, 94, 49-54. [CrossRef]

21. Nichol, G.; Cobb, L.A.; Yin, L.; Maynard, C.; Olsufka, M.; Larsen, J.; McCoy, A.M.; Sayre, M.R. Briefer activation time is associated with better outcomes after out-of-hospital cardiac arrest. Resuscitation 2016, 107, 139-144. [CrossRef] [PubMed]

22. Park, H.A.; Ahn, K.O.; Lee, E.J.; Park, J.O.; On Behalf of the Korean Cardiac Arrest Research Consortium (Ko CI). Association between Survival and Time of On-Scene Resuscitation in Refractory Out-of-Hospital Cardiac Arrest: A Cross-Sectional Retrospective Study. Int. J. Environ. Res. Public Health 2021, 18, 496. [CrossRef] [PubMed]

23. Riva, G.; Jonsson, M.; Ringh, M.; Claesson, A.; Djärv, T.; Forsberg, S.; Nordberg, P.; Rubertsson, S.; Rawshani, A.; Nord, A.; et al. Survival after dispatcher-assisted cardiopulmonary resuscitation in out-of-hospital cardiac arrest. Resuscitation 2020, 157, 195-201. [CrossRef] [PubMed]

24. Lee, Y.J.; Song, K.J.; Shin, S.D.; Lee, S.C.; Lee, E.J.; Ro, Y.S.; Ahn, K.O. Dispatcher-Assisted Cardiopulmonary Resuscitation Program and Outcomes after Pediatric Out-of-Hospital Cardiac Arrest. Pediatr. Emerg. Care 2019, 35, 561-567. [CrossRef]

25. Lee, S.Y.; Hong, K.J.; Shin, S.D.; Ro, Y.S.; Song, K.J.; Park, J.H.; Kong, S.Y.; Kim, T.H.; Lee, S.C. The effect of dispatcher-assisted cardiopulmonary resuscitation on early defibrillation and return of spontaneous circulation with survival. Resuscitation 2019, 135, 21-29. [CrossRef]

26. Siman-Tov, M.; Strugo, R.; Podolsky, T.; Rosenblat, I.; Blushtein, O. Impact of dispatcher assisted CPR on ROSC rates: A National Cohort Study. Am. J. Emerg. Med. 2020. [CrossRef]

27. Czapla, M.; Zielińska, M.; Kubica-Cielińska, A.; Diakowska, D.; Quinn, T.; Karniej, P. Factors associated with return of spontaneous circulation after out-of-hospital cardiac arrest in Poland: A one-year retrospective study. BMC Cardiovasc. Disord. 2020, 20, 288. [CrossRef]

28. Kim, J.; Kim, Y.J.; Han, S.; Choi, H.J.; Moon, H.; Kim, G. Effect of Prehospital Epinephrine on Outcomes of Out-of-Hospital Cardiac Arrest: A Bayesian Network Approach. Emerg. Med. Int. 2020, 2020, 8057106. [CrossRef]

29. Perkins, G.D.; Ji, C.; Deakin, C.D.; Quinn, T.; Nolan, J.P.; Scomparin, C.; Regan, S.; Long, J.; Slowther, A.; Pocock, H.; et al. A Randomized Trial of Epinephrine in Out-of-Hospital Cardiac Arrest. N. Engl. J. Med. 2018, 379, 711-721. [CrossRef]

30. Hanuschak, T.A.; Peng, Y.; Day, A.; Morrison, L.J.; Zhan, C.C.; Brooks, S.C.; Rescu Investigators. Patient and hospital factors predict use of coronary angiography in out-of-hospital cardiac arrest patients. Resuscitation 2019, 138, 182-189. [CrossRef]

31. Zhu, N.; Chen, Q.; Jiang, Z.; Liao, F.; Kou, B.; Tang, H.; Zhou, M. A meta-analysis of the resuscitative effects of mechanical and manual chest compression in out-of-hospital cardiac arrest patients. Crit. Care 2019, 23, 100. [CrossRef]

32. Ogawa, Y.; Shiozaki, T.; Hirose, T.; Ohnishi, M.; Nakamori, Y.; Ogura, H.; Shimazu, T. Load-distributing-band cardiopulmonary resuscitation for out-of-hospital cardiac arrest increases regional cerebral oxygenation: A single-center prospective pilot study. Scand. J. Trauma Resusc. Emerg. Med. 2015, 23, 99. [CrossRef]

33. Axelsson, C.; Herrera, M.J.; Fredriksson, M.; Lindqvist, J.; Herlitz, J. Implementation of mechanical chest compression in out-of-hospital cardiac arrest in an emergency medical service system. Am. J. Emerg. Med. 2013, 31, 1196-1200. [CrossRef]

34. Huang, J.-B.; Lee, K.-H.; Ho, Y.-N.; Tsai, M.-T.; Wu, W.-T.; Cheng, F.-J. Association between prehospital prognostic factors on out-of-hospital cardiac arrest in different age groups. BMC Emerg. Med. 2021, 21, 3. [CrossRef] 